\title{
A semi-automated line tracing technique for monitoring ice margins in Antarctic images.
}

A. Collings, R. N. Williams, N. Young and G. Hyland.

Short Title: Techniques for tracing ice margins in Antarctic images. 


\title{
A semi-automated line tracing technique for monitoring ice margins in
}

\section{Antarctic images.}

\begin{abstract}
.
The current shape and rate of change of the margins of the Antarctic ice sheet are poorly known. Since polar regions are inhospitable, remotely sensed images provide an invaluable data source for studying the ice margin. These images can be examined by a human expert or processed automatically by a computer. This paper describes the design and implementation of a semi-automated technique to trace ice margins in synthetic-aperture-radar images.
\end{abstract}

The technique is based on a deformable contour model derived from the active contour model of Kass et.al. (1988) and the discrete dynamic contour of Lobregt \& Viergever (1995). The contour is initialised by the user, to approximate the margin, and the deformation process causes the contour to accurately mold itself to the shape of the margin.

The technique has been tested on a number of different images with acceptable results in most cases.

\section{A. COLLINGS}

School of Computing, University of Tasmania, Private Bag 252, Launceston

Tasmania, Australia, 7250

R.N.WILLIAMS (corresponding author)

School of Computing, University of Tasmania, Private Bag 100, Hobart

Tasmania, Australia, $7250 \quad$ Phone: $+61(3) 63243480 \quad$ Fax: $+61(3) 63243368$

Email: R.Williams@utas.edu.au 


\section{N.YOUNG}

Antarctic Climate and Ecosystems CRC,

University of Tasmania,

Private Bag 80, Hobart, Tasmania, Australia, 7001

\section{G.HYLAND}

Antarctic Climate and Ecosystems CRC,

University of Tasmania,

Private Bag 80, Hobart, Tasmania, Australia, 7001

\section{Introduction.}

Little is known about the margins of the Antarctic ice sheet. Its shape has not been well defined and it is currently not known whether the sheet is growing or shrinking. The accuracy of current estimates of its mass balance has been given at between $10 \%$ and $50 \%$ (Massom 1991). One of the reasons for this lack of knowledge is the inaccessibility of the region.

Satellite remote sensing is a useful tool for studying regions which are difficult to visit. Images can be acquired and then studied by human experts, or processed using computers. Synthetic Aperture Radar (SAR) is ideal for observing the Antarctic region because it can operate in darkness and can penetrate cloud.

Delineation of the ice margins is an essential pre-requisite for accurately modelling changes in the overall volume of the ice sheet (Massom, 1991). This work is currently being done by 
accumulating large numbers of SAR images depicting the ice sheet margin and then manually tracing the margin in each image using a mouse. This is a time-consuming process and requires significant skill to achieve high accuracy.

A semi-automated method using artificial intelligence and image analysis techniques would allow the task of tracing the ice margin to be performed by the computer, after an initial rough approximation has been specified manually.

\section{Monitoring the ice sheet.}

\subsection{Polar ice sheets.}

The polar regions of the earth have a significant impact on global climate. Both Antarctica and Greenland are covered by vast ice sheets, These constitute $10 \%$ of the surface of the Earth and the high albedos, thermal properties and radiative properties of these areas make them climatically very significant (Massom 1991).

Seventy-five percent of the world's fresh water supplies can be found in the Antarctic ice sheet. Changes in the mass of this ice sheet cause changes in global sea level. During the last 50 years, temperatures in the Antarctic Peninsular region have increased by at least $2.5 \mathrm{deg}$

C. This has been determined from meteorological and oceanographic records. The cause of this warming is currently uncertain but it is likely that the Antarctic region will serve as a sensitive indicator for global changes in the climate in the future. This warming has caused partial disintegration of several small ice shelves along the Antarctic Peninsular. Larger ice 
shelves further south may also break up if this warming trend continues and the affected areas expand (Priddle 1999).

Although these areas are very significant, they are the least explored and most poorly understood regions of the world. Antarctica, in particular, remains largely unexplored. It is difficult and expensive to travel to Antarctica to collect measurements and make observations. This difficulty makes polar observation an ideal application for satellite remote sensing.

\subsection{The ice sheet margin.}

Since the Antarctic ice sheet has such a significant impact on climate, it is important to monitor its growth and shrinkage over time. Tracing the margins and grounding lines of the ice sheets and shelves, as well as measuring their slope, topography and elevation are all important because they serve as inputs for modelling the changes in the volume of the ice sheet.

The shape of the Antarctic ice sheet has been poorly defined in the past and it is currently not known whether the sheet is growing or shrinking. It is vital that a greater understanding is developed of the changes in the mass balance of the ice sheet, especially with current concerns related to global warming.

2.3 Monitoring the margin. 
Changes in the Antarctic ice sheet have been surveyed for a period of over fifteen years by J.Ferrigno and B.Lucchitta using archived Landsat images (Young 1996) and this work should result in an estimate of the average rate of change of the ice margin over that interval.

Young et al. (1998) have surveyed icebergs in the seasonal sea-ice zone of East Antarctica, using SAR images from the ERS-1 satellite. A semi-automated image analysis technique developed by Williams et al. (1999) was used in this survey. More recent work by Lockhart (1997) and ,Chamley (1998) focussed on matching these icebergs across multiple images to track ocean currents and to study the effects of warmer water temperatures on ice masses.

The staff of the Antarctic Climate and Ecosystems CRC in Hobart, Tasmania have been tracing the margins of the Antarctic ice sheet manually. This work was performed on SAR images from the ERS-1 and Radarsat satellites and the margins were traced by drawing over them with a mouse. An image analysis package samples points along the contour and stores them in vector format. It is hoped that the semi-automated technique developed in this research may be used for this work in the future (G.Hyland, personal communication, 1999).

\section{Line tracing techniques.}

\subsection{Active contour line tracing models.}

Line tracing is an important image analysis procedure with applications in areas such as medical imaging, remote sensing and computer vision. Line tracing can be used to identify 
the border of a feature or object in an image or it can be used to identify and characterise linear structures, such as roads, rivers or geological lineaments. In images of glaciers, line tracing could also be used to identify crevasses and flow lines produced by glacier movement.

An active contour line tracing technique begins with a coherent line structure, or contour, which roughly approximates the position of the margin being traced. It then modifies this structure so that it molds itself accurately to the shape of the margin. Such a contour maintains itself as an unbroken line structure and so is unaffected by small gaps between the original edge pixels along the margin.

\subsection{The snake model.}

The snake is an active contour model first developed by Kass, Witkin and Terzopoulos (1987). As with other active contour models, it is not intended to solve the entire problem of locating edges in images. It is expected that a higher level process, or a user, will initially position the snake near to the desired edge, after which the active contour process will cause the snake to be attracted by the edge feature.

The snake can be defined as an energy-minimising spline. In this context, the term energy is used as an analogy to the concept of energy in physics. The energy of the snake will depend on its location in the image. The desired properties in the image are represented by local energy minima in the image. A snake is always attempting to minimise its overall energy. When a snake's energy has reached a minimum, it should be resting on the image feature as desired (Sonka, Hlavac and Boyle 1995). 
There are two different forces operating on a snake; external image forces, which push the snake towards specific image features, such as edges, and internal forces, which are responsible for keeping the snake intact and maintaining the desired degree of smoothness.

A snake can be thought of as a number $(\mathbf{k})$ of discrete interconnected points, with the $\mathbf{i}^{\text {th }}$ point represented by a coordinate pair $(\mathbf{x}(\mathbf{i}), \mathbf{y}(\mathbf{i}))$. Each point has its own energy and the energy of the snake is the sum total of the energy of all the points on the snake (figure 1). Taking into account the two energy components of interest to us, we can represent the energy of the snake as follows :-

$$
E_{\text {sote }}=\sum_{i=1}^{k}\left(E_{\text {ext }} \text { (i) }+E_{\text {int }}^{\text {(i) })}\right.
$$

where $\mathbf{E}_{\text {ext }}$ is the external energy which attracts the snake to image features, and $\mathbf{E}_{\text {int }}$ is the internal energy of the snake due to bending.

$<<<* *$ Insert figure 1 approximately here $* * *>>>$

To make the snake useful it needs an external energy function, $\mathbf{E}_{\text {ext }}$ which attracts it to features in the image such as edges. The simplest image function is one which uses the

$$
E_{\text {ext }}=I(x, y)
$$

intensity (I) of the image itself, that is :- 
As it stands, minimising this energy function would attract the snake to darker regions of a light image (eg. a dark line crossing a white background). Alternatively, if we wished to attract the snake to lighter regions in a dark image, we would change the sign of $\mathbf{I}$. In many cases, however, the aim is to attract the snake to edges in an image and such edges are characterised not by high values of $\mathbf{I}$ but instead by high values of the gradient of $\mathbf{I}$ ( ie. $\boldsymbol{\nabla} \mathbf{I}$ ). This means that to attract a snake to edges in an image, the external energy is often given by the following formula:-

$$
\mathbf{E}_{\text {ext }}=|\nabla \mathbf{I}(\mathbf{x}, \mathbf{y})|^{2}
$$

where $\boldsymbol{\nabla} \mathbf{I}$ refers to the gradient of the image intensity at that point. Various approximations to the gradient of image intensity at a point can be given by different edge detectors, such as the Roberts and Sobel edge detectors.

The internal energy, $\mathbf{E}_{\text {int }}$, can consist of an elasticity component which will cause the snake to shrink around a feature of interest and will ensure that the it retains a degree of smoothness, which is characteristic of a spline. In order to achieve this behaviour, the energy needs to be defined in such a way that it increases with the length of the snake. As the snake seeks to minimise its energy, it will choose a shape with as small a length as possible. A convenient function which satisfies this condition is the sum of the squares of the distances between adjacent points.

Snakes have proven to be very useful tools, and have been used in a number of image analysis roles. They can delineate lineal structures and borders of objects in images and have 
also been useful for stereo matching pairs of images to determine the location of objects in three dimensions, as well as tracking objects in motion using sequences of digital video frames. They have been used by several researchers working in the area of medical imaging. For example Mikic, Krucinski and Thomas (1998) have used snakes to track the movement of cardiac structures in ultrasound image sequences.

\subsection{The discrete dynamic contour model.}

The discrete dynamic contour model (Lobregt and Viergever 1995) is an active contour model defined by a number of discrete points or vertices, connected by straight lines. This model is deformed by local movement of these vertices, as influenced by forces or force fields, rather than by minimising overall energy functions. The discrete dynamic contour is similar to the snake from the point of view of the user, but has a computational advantage over the snake in that rather than solving complex differential equations to minimise the overall energy of the snake, the calculations are simple and based on local deformation of vertices.

The deformation process for this model is divided into discrete steps. At the end of each step, external and internal forces are used to calculate acceleration for each vertex. This acceleration results in a change of velocity, which in turn determines the displacement of each vertex for the next step. After a number of steps, a state of equilibrium is achieved where acceleration and velocity are both zero. This represents a local energy minimum. Figure 2 shows the structure of the model. As can be seen from the diagram, the contour is made up of discrete vertices joined by straight lines. The $\mathbf{i}^{\text {th }}$ vertex is represented by $\mathbf{V}_{\mathbf{i}}$ and 
its position is represented by the vector $\mathbf{p}_{\mathbf{i}}$ (not pictured on the diagram). The vector $\mathbf{d}_{\mathbf{i}}$ represents the edge between vertices $\mathbf{V}_{\mathbf{i}}$ and $\mathbf{V}_{\mathbf{i}+\mathbf{1}}$. The forces acting on vertex $\mathbf{V}_{\mathbf{i}}$ affect its acceleration, denoted by the vector $\mathbf{a}_{\mathbf{i}}$, and therefore its velocity, given by $\mathbf{V}_{\mathbf{i}}$ (not pictured on the diagram).

$<<* * *$ Insert figure 2 approximately here $* * *>>>$

As with the snake model, the contour is affected by external image forces and internal forces. In order to calculate the external forces acting on the vertices, an external energy distribution is generated. This is effectively a new image, derived from the original image, where the desired features have low pixel values while other areas have high values. If the values of the pixels in this derived image are used as energy values then, since the dynamic contour process is attempting to minimise the energy of each vertex, the contour will move onto the desired feature.

The derived image values used in the testing of the dynamic contour were simple ones, such as original pixel values or gradient values, because they were intended to be general rather than specific to an application. The external energy force $\mathbf{f}$ ext is given by :-

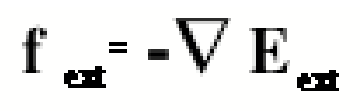

where $\nabla$ here represents the gradient of the derived image. This gradient can be measured using a range of possible edge detection techniques, such as the Sobel or Roberts edge detectors. Applying this method, however, results in a problem. The force $\mathbf{f}_{\text {ext }}\left(\mathbf{V}_{\mathbf{i}}\right)$ which 
acts on vertex $\mathbf{V}_{\mathbf{i}}$ will generally have two components. The first is perpendicular to the local direction of the contour and is referred to as the locally radial component. The second is a component along the path of the contour, referred to as the locally tangential component. The result of the tangential component is that, without internal forces, the vertices can move along the path of the contour and cluster together in areas of local minima. This problem was solved by rejecting the tangential component of the image force and only using the radial component.

Like the snake model, the discrete dynamic contour also uses internal forces to regulate the deformation by reducing the curvature of the contour, therefore smoothing it out.

The vertices in the contour are moved according to a total force $\mathbf{f}_{\mathbf{i}}$, which is a weighted combination of both external and internal forces. $\mathbf{f}_{\mathbf{i}}$ is given by :-

$$
\mathbf{f}_{\mathrm{i}}=\mathbf{w}_{\text {ext }} \mathbf{f}_{\text {ext,ri }}+\mathbf{w}_{\text {int }} \mathbf{f}_{\text {int }}
$$

where $\mathbf{f}_{\text {ext,ri }}$ is the external force in the radial direction to the vertex $V_{\mathrm{i}}$ and $\mathbf{f}_{\text {int }}$ is the internal force of the vertex $\mathbf{V}_{\mathbf{i}}$. Changing the weights $\mathbf{W}$ ext and $\mathbf{W}_{\text {int }}$ will change the behaviour of the contour. Increasing $\mathbf{W}$ ext will allow the contour to more closely follow image features, while increasing $\mathbf{W}$ int will smooth out the contour. 
The resultant effect of these forces will be a change in the vertex's position vector

During the deformation process the state of the entire contour is calculated at discrete points in time. Given values of $\mathbf{p}_{\mathbf{i}}, \mathbf{V}_{\mathbf{I}}, \mathbf{a}_{\mathbf{i}}$ and $\mathbf{f}_{\mathbf{i}}$ for vertex $\mathbf{V}_{\mathbf{i}}$ at time $\mathbf{t}$, then the corresponding values of $\mathbf{p}_{\mathbf{i}}, \mathbf{V}_{\mathbf{i}}$ and $\mathbf{a}_{\mathbf{i}}$ at time $\Delta \mathbf{t}$ can be calculated using Newton's Equations of Motion (Lobregt and Viergever 1995).

The distance between a vertex and its next neighbour represents the local contour resolution. If this is too large, the contour will not be able to follow small variations in the desired feature because they will pass through the gaps between vertices. The local resolution is always changing as the contour deforms and although this is not desirable, it is important that the vertices have the freedom to move in relation to their neighbours in order to follow the feature as closely as possible.

To resolve this conflict, the discrete dynamic contour model uses a re-sampling technique to add or remove vertices as needed, to maintain contour resolution, while still allowing the vertices freedom to move. The desired length of the edges between vertices is represented by the parameter $\mathbf{I}_{\mathbf{d e s}^{\bullet}} \quad$ Two other values, $\mathbf{I}_{\min }$ and $\mathbf{I}_{\max }$ represent the maximum and minimum lengths between vertices respectively and are given by :-

$$
l_{\min }=(1 / 2) l_{\text {des }} \quad l_{\text {max }}=\left({ }^{3} / 2\right) l_{\text {des }}
$$

The re-sampling is a two-pass process. The first pass searches along the contour for any distances less than $\mathbf{I}$ min. For each one found, the two vertices on either side of the edge are 
combined into a single vertex in the middle of the edge. The second pass searches along the contour for any edges of length greater than $\mathbf{I}_{\text {max }}$. For each one found, a new vertex is added at the mid-point between the two vertices at either end of the edge. When a new vertex is added, its acceleration and velocity are calculated by averaging the corresponding values for the vertices that it is replacing, or those between which it has been inserted.

The discrete dynamic contour has been successfully used by Lefebvre, Genevieve and Laugier (1998) to automatically detect bone boundaries in ultrasound images. They chose this approach because it was relatively simple and had been successfully used on various medical images, including ultrasound and computed tomography bone images, previously. It could be traced step by step and vertex by vertex and the number of parameters was low with the effect of each parameter being easily explained. Also the problems of contour shrinkage and vertex clustering had been solved.

\subsection{Calculation of gradients.}

An essential element of all active contour techniques is the process of finding the gradient of an image in the immediate vicinity of a specified pixel. Such a process is used to calculate the image forces which guide the deformable contour. Typically, gradient calculations use a 3 x 3 pixel mask, which we will label as follows :-

$\begin{array}{lll}\mathbf{x}_{1} & \mathbf{x}_{2} & \mathbf{x}_{3} \\ \mathbf{x}_{4} & \mathbf{x}_{5} & \mathbf{x}_{6}\end{array}$




$$
\begin{array}{lll}
\mathbf{X}_{7} & \mathbf{X}_{\mathbf{8}} & \mathbf{x}_{\mathbf{9}}
\end{array}
$$

For each pixel in the original image, a $3 \times 3$ mask is used to examine the values of surrounding pixels. This results in a slope value that is stored as a new pixel value.

A popular, but simple, method of calculating gradient is to use the two Sobel operators. One generates a gradient value, $\mathbf{G}_{\mathbf{x}}$, in the $\mathbf{X}$ (horizontal) direction, using the formula :-

$$
G_{x}=\left(x_{3}+2 x_{6}+x_{9}-x_{1}-2 x_{4}-x_{7}\right) / 9
$$

while the other generates a gradient value, $\mathbf{G}_{\mathbf{y}}$, in the $\mathbf{y}$ (vertical) direction, using the formula :-

$$
G_{y}=\left(x_{1}+2 x_{2}+x_{3}-x_{7}-2 x_{8}-x_{9}\right) / 9
$$

These values can be combined to give the magnitude of the gradient $\mathbf{G}$ using the formula :-

$$
\mathbf{G}=\operatorname{sqrt}\left(\mathbf{G}_{\mathrm{x}}{ }^{2}+\mathbf{G}_{\mathrm{y}}{ }^{2}\right)
$$

as well as its direction, using the formula :-

$$
\operatorname{dirn} \mathbf{G}=\arctan \left(\mathbf{G}_{\mathbf{x}} / \mathbf{G}_{\mathbf{y}}\right)
$$


The calculations made by the Sobel operators are simple so the operators are fast computationally.

While the Sobel operators, in common with most gradient-estimation techniques, average the values of surrounding pixels in order to arrive at a slope estimate, the maximum slope method (Dunn and Hickey 1998) does not perform an averaging operation. Instead it compares the central pixel with each of its eight neighbours. The maximum of the absolute difference between the central pixel and its neighbours is used as the gradient estimate, $\mathbf{G}$. That is :-

\section{$\mathbf{G}=\max \left|x_{5}-x_{i}\right|$}

This gradient estimate is simpler to calculate than the one using the Sobel operators, and is able to respond to very local variations in slope within the image, but it can be severely affected by image noise.

\section{The ice margin tracing technique.}

\subsection{Overall description of the technique.}

The technique developed for this work makes use of an active contour model based on the discrete dynamic contour model of Lobregt and Viergever (1995), but initially inspired by the snake model of Kass et al. (1987). It comprises four steps :- 
Step 1: The ice margin in any image will be a region of the image in which values of adjacent pixels are likely to be very different from each other; bright on the ice shelf to dark in the adjacent water. Thus they can be characterised by a high degree of variation, or heterogeneity, in pixel values. The technique, used here to measure the degree of heterogeneity within the image, is the sigma-on-mu filter (Sephton et al. 1994). When this filter is passed over a synthetic-aperture-radar (SAR) image it produces a derived image whose pixel values measure the degree of heterogeneity in the immediate vicinity of the corresponding pixels in the SAR image. The ice margin will appear in this derived image as a bright white line, while large masses of ice shelf or water will appear black. This image is used, by the subsequent active contour technique, as an external energy field.

Step 2: This step involves placing the active contour in its initial location, ready for deformation to begin. The user enters the main points through which the initial contour will pass by clicking with the mouse on the image. These points are placed on the contour and serve as an initial approximation of the edge. After this, a re-sampling algorithm is run over the contour to insert a set of intermediate vertices.

Step 3: After its initial position has been defined, the contour is then activated. The active contour technique employed here represents the contour as a collection of discrete vertices, in which each vertex moves in the direction of the local gradient in total energy in the immediate vicinity of the vertex. This total energy value includes external energy, represented by image features, and internal energy, represented by the stiffness constraints imposed on the contour itself. Before the contour is activated, the user is given the option to change a number of parameters, such as stiffness, to suit the situation. Once the parameters have been set, the deformation process is begun. After the contour has reached an 
equilibrium position, the user has the opportunity to accept it or to change some of the parameters and continue deformation.

Step 4: After the final contour has been obtained, it can be saved as a vector representation suitable for importing into a geographic information system or some other remote sensing data analysis package.

\subsection{Image preprocessing.}

The SAR images used in this study were generated by the European Space Agency (ESA) as SAR Precision Image products (ERS.SAR.PRI). They are 3-look, ground-range images which have been corrected for geometric and instrumental factors. The image data have a pixel size of $12.5 \mathrm{~m}$ by $12.5 \mathrm{~m}$ and have a potentially high spatial resolution, nominally $30 \mathrm{~m}$, but the high level of speckle inherent in 3-look imagery limits the size of the objects that can be resolved using the radiometric information. In order to reduce this speckle to an acceptable level, the images were smoothed by an 8 x 8 pixel block-averaging process, giving a pixel size of $100 \mathrm{~m}$ by $100 \mathrm{~m}$ and an effective number of looks of 30 . This significantly reduced the speckle of the mean signal level for homogeneous areas but at the expense of degrading the spatial resolution.

The process of defining the ice margin within the image begins with determining which parts of the image exhibit significant variation and which are essentially homogeneous. The detection of heterogeneous regions can be carried out by scanning the image with an edge detection operator. A wide variety of such operators could be used to do this. However, a 
simple statistically-based edge detector, the sigma-on-mu filter (Williams et al. 1999, Sephton et al. 1994) has proven effective for this work. The detector scans the image using a $3 \times 3$ pixel window and calculates the standard deviation $(\sigma)$ and mean $(\mu)$ of the intensity values of the nine pixels within the window. A new image is generated from the values of $\sigma / \mu$ corresponding to each pixel. For images containing multiplicative noise, such as SAR images, the expectation value of this ratio for a homogeneous region is independent of mean intensity. However, when the window passes over the boundary between two regions with differing mean intensity, the variation in pixel value is greatly increased, thus increasing the value of $\sigma / \mu$. This filter is sensitive to the presence of an edge irrespective of its orientation in the image and is considered particularly suitable for SAR images because it caters for the multiplicative nature of the speckle noise that occurs in such images (Sephton et al. 1994).

The result of the sigma-on-mu edge detection process is a derived image which represents the degree of variation in the immediate vicinity of each pixel in the speckle-reduced SAR image. If this image is then used to represent the image energy for an active contour deformation process, the active contour will be attracted to an energy maximum represented by those pixels in which the variation in the immediate pixel neighbourhood is a maximum; ie. the precise ice margin.

4.2 The deformation process. 
The deformation process used to move the contour into its final position alongside the ice margin involves moving each vertex separately in the appropriate direction according to external and internal energy forces. Several different mechanisms have been used to move the vertices in response to these forces and these mechanisms are described below. These various mechanisms have been employed in the overall deformation process according to the characteristics of the image being processed, the requirements of the user and the stage of the deformation process being carried out. These mechanisms have been modelled on those introduced by Kass et al. (1987) and Lobregt and Viegever (1995) but have been modified to reduce their computational requirements for this task.

\subsection{Moving a vertex in response to external forces.}

Two different techniques were used to move the vertex in response to external forces. The first compares the energy (in our case the sigma-on-mu value) of the current vertex pixel with that of the all of the immediately surrounding pixels. It determines the maximum energy value and moves the vertex to the pixel with the maximum value. This approach is similar to the non-averaging slope method, the Maximum Slope Method, described by Dunn and Hickey (1998). If the current vertex position has the highest energy value, the vertex is not moved. This technique was modified by enabling it to look up to 3 pixels away in any of eight directions (up, down, left, right, up-left, up-right, down-left and down-right) and then move the vertex to the pixel position which corresponds to the maximum energy of all of these pixels. 
The second technique is based on the use of Sobel edge detection operators. Rather than using the two Sobel operators together to calculate a magnitude and direction for the image gradient in the immediate vicinity of the vertex (requiring a computationally demanding arctan calculation as described in section 3.4), the direction of vertex movement is implied from the $\mathrm{x}$ and $\mathrm{y}$ Sobel values. For example if the $\mathrm{y}$ Sobel operator generated a positive value above a specified threshold and the $\mathrm{x}$ Sobel operator also generated a value above the threshold, then the vertex was moved one pixel in the up-right direction. Other directions were implied according to the respective signs of the $\mathrm{x}$ - and $\mathrm{y}$-Sobel operators. If neither operator generated a value above the threshold, the vertex was not moved.

\subsection{Moving a vertex in response to internal forces.}

The internal energy term used in the contour deformation process is a discrete version of the elasticity term of the original Snake Model (Kass et al. 1987). An internal energy value is calculated for each of the eight directions in which the vertex can move. In order to calculate this, the algorithm checks both neighbouring vertices. If the neighbour being examined is further advanced in the current direction (eg. while examining the up direction, if the neighbour is above the current vertex) then some internal energy is added to pull the current vertex with it. The amount of energy added $(\Delta \mathbf{E})$ is proportional to the distance between the vertex and its neighbour, $\mathbf{d}$ vertex,neighbour, and the value of the elasticity parameter (referred to as $\boldsymbol{\alpha}$ in the Snake Model), ie. :-

$$
\Delta \mathbf{E}=\mathbf{d}_{\text {vertex, neighbour }} * \alpha
$$


This is similar to the equation for calculating elasticity in the snake model given by Young (1995). If $\boldsymbol{\alpha}$ is zero, there will be no internal energy and the contour will be guided by external image forces alone. The elasticity constraint is useful in the deformation process in two ways. If most of the vertices within a contour are being attracted towards a specific image feature but one or two are not because of local image anomalies, these vertices will be attracted by their neighbours and so will be drawn into the influence of the image feature of interest. Also the elasticity constraint can prevent the formation of very convoluted contour shapes, to which the discrete dynamic contour model is susceptible.

\subsection{The overall technique.}

The ice margin tracing technique described above was implemented using the Interactive Data Language (IDL) written by Research Systems Inc. It was developed as a subsystem of an existing program called SARTRACKER, which had been written to identify and delineate icebergs within SAR images (Williams et al. 1999). The SARTRACKER program now incorporates a control panel which can be used to invoke the margin tracing routines and via which the user can set certain parameters related to the deformation process being used. This can be used to fine-tune the settings to the particular application, but has also been used to experimentally determine how to obtain the desired behaviour from the contour.

All of the parameters can be changed before the deformation begins, but they can be further altered at the end of each deformation cycle (ie. after 30 steps). This means that, for example, the external energy calculation method can be changed from the Sobel method to the pixel brightness method part way through the deformation process. The two main parameters 
within the contour deformation process, elasticity $(\boldsymbol{\alpha})$ and desired vertex distance $\left(\mathbf{I}_{\mathbf{d e s}}\right)$, can also be modified via the control panel. Also, the degree of prior smoothing of the sigmaon-mu image can be set at $3 \times 3,9 \times 9$ or $15 \times 15$. Setting this to a larger value makes the active contour sensitive to the presence of the ice margin from a greater distance away, while setting it to a smaller value enables the contour to mold itself to the precise shape of the ice margin, provided that the initial contour was already quite close to the margin in the first place.

It was found that no one set of parameters was suitable for all situations and no method of external energy calculation was suitable for all examples. The pixel brightness technique had the advantage of positioning the contour along the margin with high precision but had the disadvantage of needing to start close to the margin to begin with and being susceptible to image noise. On the other hand, the modified Sobel operator method was able to attract the contour to the margin from quite a distance using a smoothed sigma-on-mu image and was less susceptible to image noise, but the effect of the smoothing process was that detailed shape information on the ice margin was lost.

Since no method was suitable for all situations, it became apparent that the best approach was to use multiple stages and to change the method part way through. This type of approach was used by Wang et al. (1996) in the development of their multi-stage optimal snake. As in Wang's model, three different stages were identified :-

(a) The broad stage: The purpose of this stage was to bring the vertices of the contour close enough to the ice margin to be shaped by later stages. It used the $15 \times 15$ smoothed sigma- 
on-mu image and the modified Sobel method for external energy calculation and used an elasticity value of 5 as a default, although this could be changed by the user if desired.

(b) The shaping stage: The purpose of this stage was to bring the contour closer to the ice margin and to begin to shape it. It used a $9 \times 9$ smoothed sigma-on-mu image and the modified Sobel method for external energy calculation. At the end of this stage, the contour should roughly follow the shape of the ice margin.

(c) The fine-tuning stage: The purpose of this stage was to fine-tune the contour so that it followed the ice margin as closely as possible. The best option for this stage was found to be the pixel brightness method, applied to the sharp sigma-on-mu image. This method moved all of the vertices toward the brightest nearby pixel, which had the effect of fitting the contour very closely to the ice margin. The pixel-brightness method did not need to use as high an elasticity as the modified Sobel method and so a value of 2 was set as a default.

In the final operational version of this software, options will be made available to the user to invoke each of these three stages on demand. Ideally, the change from one stage to another should be fully automatic. The obvious point for this to occur would be the point at which the current stage reaches equilibrium. The major problem with this is that the contour often fails to reach a single equilibrium configuration but continues fluctuating slightly between the pull of the external and internal forces. It may be possible to solve this problem by fully implementing the force, velocity, acceleration model of Lobregt and Viergever (1995) because this would allow a damping force to be incorporated into the model. 


\section{Performance evaluation.}

5.1. Test data.

The ice margin tracing technique was tested on segments taken from five different SAR images, with results from three of these images described here. These image segments were chosen to assess the performance of the technique under increasingly difficult conditions. All images were generated by the European Space Agency (ESA) as SAR Precision Image products (ERS.SAR.PRI) and subsequently smoothed by an 8 × 8 pixel block-averaging process to reduce the speckle within the image. The final images had a pixel size of $100 \mathrm{~m} \mathrm{x}$ 100m. The details of the images are as follows :-

Image ID Acquisition Date and Time Location of Image Centre
e1_10755_5013_av8.dat 6-Aug-93 03:12:20
$67.86 \operatorname{deg} \mathrm{S} 79.74 \operatorname{deg} \mathrm{E}$
e1_10865_5787_av8.dat 13-Aug-93 19:49:02
$67.64 \operatorname{deg} \mathrm{S} 69.63 \mathrm{deg} \mathrm{E}$
e1_10755_4995_av8.dat 6-Aug-93 03:12:05
$67.09 \operatorname{deg} \mathrm{S} 81.01 \mathrm{deg} \mathrm{E}$

These images were used to define four different regions which were used to test the ice margin tracing technique under increasingly difficult conditions. The regions were labelled as follows :-
Region number
Image from which region was extracted.
region 1
e1_10755_5013_av8.dat 


$\begin{array}{ll}\text { region } 2 & \text { e1_10865_5787_av8.dat } \\ \text { region } 3 & \text { e1_10865_5787_av8.dat } \\ \text { region } 4 & \text { e1_10755_4995_av8.dat }\end{array}$

\subsection{Performance on regions 1 and 2.}

The ice margin in region 1 was relatively straight, without curves or indentations (Fig 3(a)). The contour was initialised at just two points, each one near to where the ice margin reached the edge of the region. The initial and final contour positions are shown in Fig 3 (a) and (b) respectively. The contour located the ice margin to a high degree of accuracy in this case.

$<<*$ Insert Fig $3(\mathrm{a}),(\mathrm{b}),(\mathrm{c})$ and (d) approximately here $* *>>$

The ice margin in region 2 was more difficult to trace (Fig 3(c)). On the left of the region, it was not well defined, which meant that the sigma-on-mu values were relatively low. Secondly, there was significant noise in the vicinity of the margin, probably generated by backscatter from adjacent sea-ice. The margin was a relatively straight line, but it had a sharp bulge and a gentle bay which complicated the tracing process. Again, the contour was initialised with two points, one each at the endpoints of the margin on the region. The initial and final contour positions are shown in Fig 3 (c) and (d) respectively. The pixel brightness method alone had great difficulty with this example. It was severely affected by the presence of the noise pixels. However the multi-stage approach was able to locate the ice margin with a high degree accuracy, except for one section, just to the left of the bulge, where a sharp indentation was not accurately represented in the final contour position. 


\subsection{Performance on regions 3 and 4.}

The ice margin in region 3 was a large convex curve (Fig 4(a)). The contour was initialised at five points, roughly approximating the curve of the margin but placed outside the curve. The initial and final contour positions are shown in Fig 4 (a) and (b) respectively. The contour located the ice margin to a high degree of accuracy (note that the upper image in Fig 4(b) shows the ice margin as traced manually) although for values of $\boldsymbol{\alpha}$ above 1 the elasticity constraint had a tendency to cause the contour to continue shrinking past the margin.

$<<* *$ Insert Fig 4 (a), (b), (c) and (d) approximately here **>>

The ice margin in region 4 was especially challenging. It contained several sharp angles and a long narrow indentation. Complicating the issue further was an iceberg located in close proximity to the margin at one point (Fig 4(c)). This time the contour was initialised at seven points roughly approximating the margin. The initial and final contour positions are shown in Fig 4 (c) and (d) respectively. In this case the contour was able to trace the margin reasonably accurately along most of its extent but was unable to accurately represent the innermost part of the margin indentation depicted in the image. Also the result was extremely sensitive to contour initialisation and parameter values. To achieve an acceptable result, these had to be fine-tuned to a degree that would be impractical in an operational setting. 


\subsection{Summary of performance.}

The active contour technique performed well with relatively simple contours but with more complex contours the technique experienced some difficulty. If the contour was initialised sufficiently close to the margin it was able to trace the margin with a high degree of accuracy with all but the very strongly indented margin in region 4 . However in regions 3 and 4 the technique was susceptible to the position of the initial contour. Despite these inadequacies, this technique showed promise for operational ice margin tracing applications.

\subsection{Subsequent operational use of the technique.}

To investigate the possibility of developing an operational system (M.Moffew, private communication, 2001)the three stages of the technique described here (the Broad Stage, the Shaping Stage and the Fine-tuning Stage) were tested to evaluate their relative effectiveness on a specific sample of images being used for the ice margin tracing project. This work suggested that it would be possible to construct an effective tool by providing three processing options and allowing the ice margin tracer to manually change between options, during the tracing process, according the characteristics of the ice margin being traced.

An active contour method, using a Sobel gradient filter applied to a 3 x 3 smoothed sigma-on$m и$ image as the method for external energy calculation, was used as the primary ice margin tracing tool. However, the pixel brightness method was also made available as an optional fine tuning stage, after the $3 \times 3$ Sobel gradient method had been applied, in situations where the ice margin was clearly defined on the image. Finally, an alternative active contour method using a Sobel gradient filter applied to a 9 × 9 smoothed sigma-on-mu image, was 
made available to replace the technique using the $3 \times 3$ smoothed image in situations where the ice margin is relatively poorly defined.

These three options were found to be sufficient to enable semi-automated ice margin tracing in the majority of the images provided. Manual ice margin tracing was used for the remainder.

\section{Conclusion.}

A technique for the semi-automated tracing of ice margins in remotely sensed images is an essential facility required to support research being undertaken by the Antarctic Climate and Ecosystems CRC to determine the current mass-balance of the Antarctic ice sheet. The work described here has shown that an active-contour based approach has the potential to be a useful technique for enabling semi-automated ice margin tracing to be carried out (M.Moffew, private communication, 2001). The contour-based approach is particularly suitable for tracing the long, and sometimes broken, edges which represent ice margins in remotely sensed images because, unlike more locally-based line tracing techniques, it maintains the connectivity of the line at all times. This research has confirmed that an activecontour approach, which takes a user-specified initial contour, positioned in the vicinity of an ice margin, and then refines it via a deformation process until it precisely defines the margin, is an effective approach for this application.

\section{Acknowledgements.}


We thank staff from the Antarctic Climate and Ecosystems CRC, who provided assistance and support for this project and Mr Tony Gray, from the School of Computing, for his assistance in installation of the IDL remote sensing package. The research work reported in this paper was partly funded by the award of a research grant provided by the Antarctic Science Advisory Council (Project No. 1125).

\section{References.}

CHAMLEY, C., 1998, Matching icebergs in pairs of synthetic aperture radar images using shape analysis, School of Computing honours thesis, University of Tasmania, Launceston.

COHEN, L., 1991, Note on active contour models and balloons, CVGIP Image Understanding. 53, 211-218.

DUNN, M. and HICKEY, R., 1998, The effect of slope algorithms on slope estimates within a GIS. Cartography, 27, 9-15.

FAHNESTOCK, M. A., 1997, Satellite-based measurement of ice sheet surface properties and motion (ABSTRACT ONLY), Workshop on Remote Sensing of Planetary Ices: Earth and other Solid Bodies. June 11-13 1997. Flagstaff, Arizona, http://wwwflag.wr.usgs.gov/USGSFlag/Space/RSIce/rsice.html 
GUNN, S. and NIXON, M., 1997, A robust snake implementation; A dual active contour, IEEE Transactions on Pattern Analysis and Machine Intelligence, 19, 63-68.

KASS, M, WITKIN, A. and TERZOPOULOS, D., 1987, Snakes: active contour models, International Journal of Computer Vision, 1, 321-331.

LEFEBVRE, F., GENEVIÈVE, G. and LAUGIER, P., 1998, Automatic detection of the boundary of the calcaneus from ultrasound parametric image using an active contour model; clinical assessment, IEEE Transactions on Medical Imaging, 17, 45-52.

LOBREGT, S. and VIERGEVER, M., 1995, A discrete dynamic contour model, IEEE Transactions on Medical Imaging, 14, 12-24.

LOCKHART, M., 1997, A Feature Matching Technique for Tracking Icebergs in Image Sequences obtained from Synthetic Aperture Radar Images, School of Computing honours thesis, University of Tasmania, Launceston. 1997.

MASSOM, R., 1991, Satellite Remote Sensing of Polar Regions : Applications, Limitations and Data Availability (London: Bellhaven Press in association with the Scott Polar Research Institute, University of Cambridge).

MIKIC, I, KRUCINSKI, S and THOMAS, J., 1998, Segmentation and tracking in echocardiographic sequences: active contours guided by optical flow estimates, IEEE Transactions on Medical Imaging, 17, 274-284. 
PRIDDLE, J., 1999, Moving towards a synthesis of global change in the Antarctic.

Antarctic Global Change Research. Newsletter of the SCAR Global Change Programme. No 4.

SEPHTON, A., BROWN, L., MACKLIN, J., PARTINGTON, K., VECK, N. and REES, W. G., 1994, Segmentation of synthetic-aperture radar imagery of sea ice, International Journal of Remote Sensing, 15, 803-825.

SONKA, M., HLAVAC, V. and BOYLE, R., 1995, Image Processing, Analysis and Machine Vision ( London: Chapman \& Hall).

WANG, M., EVANS, J., HASSEBROOK, L. and KNAPP, C., 1996, A multistage, optimal active contour model, IEEE Transactions on Image Processing, 5, 1586-1591.

WILLIAMS R.N., REES W.G. \& YOUNG N.W., 1999, A technique for the identification and analysis of icebergs in synthetic aperture radar images of Antarctica, International Journal of Remote Sensing, 20, 3183-3199.

YOUNG, N., 1996, Iceberg Distribution and Southern Ocean Circulation. Proposal in response to NASA NRA-94-MTPE-05. Project ID 351.

YOUNG, N. W., TURNER, D., HYLAND, G. and WILLIAMS, R. N., 1998, Nearcoastal iceberg distributions in East Antarctica, 50-145 E, Annals of Glaciology, 27, 6873. 


\section{Figure captions.}

Figure 1. The snake model.

Figure 2. The discrete dynamic contour model.

Figure 3. (a) and (b): Ice margin tracing evaluation on region 1.

$\mathbf{l}_{\text {des }}=4$ for all stages.

$\boldsymbol{\alpha}=5$ for the broad and shaping stages and $\boldsymbol{\alpha}=2.2$ for the fine-tuning stage .

Initial contour position in (a). Final contour position in (b).

(c ) and (d): Ice margin tracing evaluation on region 2.

$\mathbf{l}_{\mathbf{d e s}}=4$ for all stages.

$\boldsymbol{\alpha}=5$ for the broad and shaping stages and $\boldsymbol{\alpha}=2$ for the fine-tuning stage.

Initial contour position in (c ). Final contour position in (d).

Figure 4. (a) and (b): Ice margin tracing evaluation on region 3.

$\mathbf{l}_{\text {des }}=4$ for all stages.

$\boldsymbol{\alpha}=1$ for all stages.

Initial contour position in (a).

Final contour positions in (b). The manually derived contour is shown in the upper image and the automatically derived contour is shown in the lower image.

(c) and (d): Ice margin tracing evaluation on region 4.

$\mathbf{l}_{\text {des }}=4$ for all stages.

$\boldsymbol{\alpha}=0$ for the broad and $\boldsymbol{\alpha}=1$ for the shaping stage

$\boldsymbol{\alpha}=2$ for the fine-tuning stage. 
Initial contour position in (c). Final contour position in (d). 